\title{
INTERVENÇÃO PARA A PROMOÇÃO DA ADESÃO AO REGIME \\ TERAPÊUTICO DA PESSOA EM INÍCIO DE HEMODIÁLISE: UMA \\ PESQUISA QUALITATIVA
}

\section{INTERVENTION TO PROMOTE ADHERENCE TO THERAPEUTIC \\ REGIMEN AT INITIATION OF HEMODIALYSIS: A QUALITATIVE \\ RESEARCH}

\author{
Maria Isabel Domingues Fernandes ${ }^{1}$ \\ Liliana Marlene Ferreira $\mathrm{Cruz}^{2}$
}

\begin{abstract}
Resumo: A investigação tem como objetivo analisar a intervenção da equipa de enfermagem de um serviço de Nefrologia na promoção da adesão ao regime terapêutico da pessoa com doença renal crónica em início de hemodiálise. É um estudo de caso único sustentado numa amostra de meio com colheita de dados por entrevista em grupos focais. $\mathrm{O}(\mathrm{s})$ conceito(s) de adesão dos profissionais, o processo de acolhimento, a educação e avaliação da capacitação da pessoa para iniciar hemodiálise e, os aspectos considerados na promoção da adesão descrevem/explicam a intervenção. Para promover este processo a capacitação da pessoa, envolvimento da família, regressão dos sintomas, confiança e simpatia, relação de proximidade e ganhos em saúde são fundamentais.
\end{abstract}

Palavras-chave: Regime terapêutico; Enfermagem; Hemodiálise; Adesão; Promoção.

\begin{abstract}
The study aims to analyze the intervention of the nursing team of a Nephrology unit to promote the adherence to the therapeutic regimen of patients with chronic kidney disease at initiation of hemodialysis. This is a single case study with a milieu sample and interviews in focus groups. Interventions are described/explained by professionals' concepts of adherence, process of admission, patient education, and evaluation of the patient's preparation for hemodialysis, as well as aspects related to promoting adherence. Patient empowerment, family engagement, symptom regression, trust and sympathy, relationship of proximity, and health gains are essential to the promotion of adherence.
\end{abstract}

Keywords: Therapeutic regimen; Nursing; Hemodialysis; Adherence; Promotion.

\section{Introdução}

As doenças crónicas, denominadas também como doenças não transmissíveis, pela World Health Organization (WHO, 2017, p. 5), pela sua dimensão e pelo número de mortes prematuras que ocasionam continuam "[...] to be one of the major development challenges in the 21 st century."

\footnotetext{
${ }^{1} \mathrm{PhD}$ em Didática pela Universidade de Aveiro (UA). Professora Coordenadora da Escola Superior de Enfermagem de Coimbra (ESEnfC), Coimbra, Portugal. Email: isabelf@esenfc.pt

${ }^{2}$ Mestre em Enfermagem Médico-Cirúrgica pela Escola Superior de Enfermagem de Coimbra. Enfermeira no Centro Hospitalar e Universitário de Coimbra (CHUC), Coimbra, Portugal. E-mail: lianafcruz@gmail.com
} 
A prevenção e controlo destas doenças (nomeadamente doenças respiratórias e cardiovasculares crónicas, cancro e diabetes), tendo em vista a redução para um terço das mortes prematuras, até 2030, e a promoção da saúde mental e do bem estar, foram compromissos dos líderes mundiais expressos na Agenda para o Desenvolvimento Sustentável. A monitorização do progresso da implementação do compromisso político das medidas de prevenção e controlo "[...] has been insufficient and highly uneven [...]" (WHO, 2017, p.5).

O aumento das doenças crónicas está relacionado com o progressivo envelhecimento da população, os comportamentos de risco, o fator ambiental e a diminuição da mortalidade, o que tem colocado novas necessidades e novos desafios na procura de cuidados de saúde. Esta é uma realidade inquietante, um dos maiores desafios para os profissionais de saúde e para os dirigentes políticos, tanto pelas suas repercussões ao nível da saúde populacional, como pelas consequências ao nível social e económico (WHO, 2014). O acréscimo significativo de pessoas com doença crónica é uma realidade cada vez mais expressiva quer a nível nacional quer internacional. A doença renal crónica é uma destas condições clínicas.

O aumento significativo do número de pessoas com doença renal crónica terminal (DRCT), em Portugal e no mundo, resulta numa condição de saúde que provoca elevados custos em termos humanos, sociais e económicos (DRAWZ, et al., 2012). O tratamento destas pessoas é complexo e agressivo pois, exige com frequência, o recurso a uma das técnicas de substituição da função renal. A pessoa é, assim, confrontada com um regime terapêutico multifacetado e contínuo, complexo de gerir e a implicar mudanças e adaptações nos seus hábitos de vida. A pessoa com doença renal que vai iniciar hemodiálise está particularmente vulnerável necessitando de ser ajudada neste processo. Os enfermeiros assumem uma importância fundamental em todo o processo da doença, nomeadamente na promoção da adesão ao regime terapêutico (RT).

A adesão exige a mobilização dos recursos interiores da pessoa, permitindo que integre as suas expectativas no que se prende com a saúde e vida. A participação das pessoas com doença crónica nos seus cuidados acontece a três níveis, de acordo com o modelo desenvolvimental de Adesão ao Tratamento de Kristeller e Rodin (1984), apresentado por Catela e Amendoeira (2010), a saber: concordância (compliance) - fase inicial em que a pessoa concorda e segue a prescrição; adesão (adherence) - a pessoa continua o tratamento com que concordou, mesmo quando ocorrem alterações da situação, havendo alguma vigilância do processo; manutenção (maintenance) - a pessoa 
mantém a adesão ao RT de forma autónoma, sem vigilância, no sentido de garantir o seu bem-estar e um estilo de vida saudável (CATELA; AMENDOEIRA, 2010).

Há evidência que o sucesso do tratamento depende em grande parte desta adesão a um regime estritamente recomendado e, que a não adesão, pode ter resultados negativos na qualidade de vida $(\mathrm{QV})$, no aumento da morbilidade, da mortalidade e no gasto em saúde (ALIKARI, et al., 2015).

A adesão ou a não adesão na pessoa em tratamento dialítico está dependente de múltiplos aspectos no entanto a investigação tem documentado largamente que as intervenções educativas levam a uma maior adesão, por exemplo, às restrições de fluidos e sódio (SHARAF, 2016), à dieta e ingestão de medicação (ALIKARI; FRADELOS; ZYGA, 2015). O déficit de conhecimento sobre a doença, limitações decorrentes do tratamento e alterações durante a sessão de hemodiálise têm sido associados a comportamentos de não adesão (MACIEL et al., 2015).

Estes terão estado entre os fundamentos, da recomendação da Direção Geral da Saúde (DGS, 2011) para que em cada serviço de nefrologia exista uma consulta multidisciplinar dedicada ao esclarecimento do doente ou, muito mais precocemente, a EDTNA - European Dialysis and Transplant Nurses Asssociation, definisse as competências do enfermeiro em nefrologia e realçasse a importância da implementação de consultas de enfermagem nefrológica com o objetivo da educação da pessoa sobre todo o processo associado ao tratamento (CHAMNEY, 2007).

Embora as competências dos enfermeiros de nefrologia variem muito entre países, os curricula da sua formação prevêem uma formação que os prepare para assumirem: atividades de avaliação da pessoa com DRCT, tanto a nível da resposta e necessidades físicas como psicossociais e educacionais; a coordenação da intervenção da equipa multidisciplinar; o desenvolvimento e implementação de programas de educação da pessoa; e a promoção de actividades de autocuidado (CHAMNEY, 2007). O enfermeiro na equipa, tal como apresentam Goovaerts et al., (2015), tem sob a sua responsabilidade diminuir a complexidade em torno da diálise, proporcionando e organizando a formação, gerindo a situação de cada pessoa, individualizando e avaliando o processo de educação e, dando apoio. É um cuidador chave assumindo frequentemente a função de "gestor de caso" (GOOVAERTS, et al., 2015).

Contudo, a atividade que o enfermeiro em cada dia desenvolve com a pessoa, hospitalizada para iniciar hemodialise, na ou para a adesão ao regime terapêutico, tem sido insuficientemente explorada na visão destes profissionais. Assim, a partir da reflexão 
sobre a forma como os enfermeiros de um serviço de Nefrologia intervêm no processo formativo da pessoa com doença renal crónica em início de hemodiálise e, tendo presente os requisitos e as necessidades em cuidados de enfermagem no que se refere à promoção de adesão ao regime terapêutico, procurou-se analisar a intervenção da equipa de enfermagem neste processo, com a pessoa com doença renal crónica em início de hemodialise, tendo os enfermeiros como participantes.

\section{Metodologia}

Com a investigação pretende compreender-se como intervêm os enfermeiros no processo formativo da pessoa com doença renal em início de hemodiálise, de forma a promover a adesão ao regime terapêutico. Para tal, importa conhecer e aprofundar a experiência dos enfermeiros neste âmbito, identificando estratégias de intervenção para a promoção de um trabalho qualitativamente diferenciado.

Assim, desenvolveu-se um estudo descritivo e transversal inserido numa abordagem de natureza qualitativa, partindo da questão de investigação - A intervenção da equipa de enfermagem, de um serviço de Nefrologia, é promotora do processo de adesão ao regime terapêutico da pessoa com doença renal crónica em início de hemodiálise? Definiram-se como objectivos: i) compreender como se processa o acolhimento no serviço de nefrologia e a preparação da pessoa com doença renal crónica para o início de hemodiálise; ii) analisar as estratégias/recursos utilizados na preparação da pessoa com doença renal crónica em início de hemodiálise para promover a adesão ao regime terapêutico; iii) analisar as razões subjacentes à intervenção do enfermeiro na promoção da adesão;

A amostra constituiu-se em função do conjunto de características que se pretendiam analisar, para tal na selecção dos participantes procedeu-se a uma amostragem de caso único, identificando-se uma amostra de meio ou institucional, seguindo o definido por Pires, em 2012. O autor considera que se escolhe um meio como universo de análise (e é nele que se constitui o corpus empírico) e, este apresenta-se "ao analista de forma não parcelada e como sendo passível de uma apreensão na sua totalidade" (PIRES, 2012, p. 177). Os participantes seriam todos os vinte e seis enfermeiros que integravam a equipa de enfermagem do referido serviço. Foram excluídos aqueles que, regularmente não prestassem cuidados diretos à pessoa com DRC em início de hemodiálise, os que não aceitassem participar no estudo e, os que exercessem atividade profissional no serviço há 
menos de 6 meses. Assim, participaram vinte e um enfermeiros dado que, um não cumpria os critérios de inclusão e quatro estavam ausentes nos momentos de realização da entrevista. Saliente-se que esta tinha sido previamente acordada e agendada.

O acesso ao campo e aos participantes, para a colheita de dados, obedeceu a duas formalidades: o pedido de apreciação do projeto de investigação pela Comissão de Ética da Unidade Hospitalar, que obteve parecer favorável à colheita de dados; o contacto individualizado aos participantes com a dupla finalidade de, apresentar o estudo pedindo a sua participação e consentimento e, estabelecer uma primeira abordagem para o agendamento das entrevistas (POUPART et al., 2012).

A técnica utilizada na colheita de dados foi a entrevista semiestruturada em grupos focais. A opção pela entrevista em grupo sustentou-se nalguns dos pressupostos apresentados por Barbour (2009) que identifica, a discussão do grupo e a garantia da conversação dos participantes entre si, como ponto forte desta técnica. O importante é, também para Nunes (2012), a interação entre os participantes, pelo diálogo, troca de experiências, ideias, sentimentos e dificuldades o que permite que a participação individual de cada um seja melhorada em contexto de grupo e enriquecidas as perceções e opiniões individuais.

Sustentado nos pressupostos apresentados por aqueles autores houve a oportunidade de compreender, com alguma profundidade, o comportamento do grupo acerca do tema e, a discussão e reflexão geradas terão ajudado à construção de conhecimentos dentro do próprio grupo (BARBOUR, 2009; NUNES, 2012).

Cada grupo focal foi orientado por uma moderadora (a investigadora) e um observador, um enfermeiro da unidade hospitalar, previamente preparado e familiarizado com a investigação, o método de colheita de dados e a sua função no decorrer da entrevista. Assim, os dois elementos que desenvolveram cada entrevista e, a estratégia que usaram durante a sua realização, foram cruciais para o sucesso e riqueza da informação reunida (FERNANDES; CRUZ, 2017).

As entrevistas foram agendadas de acordo com as disponibilidades de cada um dos participantes do grupo, decorreram num período extra-laboral, foram audiogravadas e decorreram numa sala da unidade de cuidados, previamente preparada, que permitiu que estas decorressem sem interrupções. As questões exploradas nas entrevistas em grupo foram previamente preparadas e reunidas num documento orientador (POUPART et al., 2012). As questões orientavam os participantes para que analisassem: os conceitos e as práticas na admissão da pessoa; a preparação da pessoa para a adesão ao regime 
terapêutico e indicadores de avaliação utilizados; e as estratégias e recursos mobilizados na educação da pessoa e/ou família.

A construção dos quatro grupos obedeceu às orientações de Carey (2007), organizando os participantes em 3 grupos de 5 elementos e 1 grupo de 6 elementos. Foi uma construção intencional e o mais homogénea possível no que se prende com o conhecimento do fenómeno em estudo. Porém com características heterogéneas entre si (idade, sexo, formação profissional, anos de atividade profissional, aptidão comunicacional, ...) por forma a favorecer a discussão, os relatos de experiências e a diversidade de opiniões. De algum modo, pretendeu-se conciliar a homogeneidade do grupo, mantendo as características da heterogeneidade e, conciliar a uniformidade com a diversidade (NUNES, 2012).

O conteúdo dos dados narrativos foi analisado seguindo os critérios propostos por Bogdan e Biklen (2013), Carey (2007) e Barbour (2009) que são congruentes entre si. A análise implicou um conjunto de etapas sequenciadas desde a organização de dados, a divisão em unidades manipuláveis, síntese, procura de padrões e descoberta dos aspetos mais significativos para a compreensão do fenómeno em estudo.

Procedeu-se a uma primeira leitura global e fluente para conhecer o corpus de análise resultante da transcrição das entrevistas audiogravadas, saliente-se que nesta foram integradas notas relativas a cada um dos grupos focais de acordo com a recomendação de Carey (2007, p. 232) que considera necessário o investigador atender ao "[...] contexto de todo o grupo, os dados não verbais e as mudanças e discrepâncias dos contributos."

Tendo como referência as questões orientadoras das entrevistas em grupo, foi desenvolvido um sistema de codificação inicial. Duas investigadoras, sendo uma a que moderou as entrevistas, independentemente, leram e codificaram cada um dos focus grupo transcritos, neste processo a partir do questionamento dos dados identificaram códigos entre os inicialmente definidos e outros novos. Num segundo momento, analisaram conjuntamente a codificação elaborada, discutindo e consensualizando as discrepâncias encontradas. O processo de análise foi concluído comparando estes códigos, analisando-os e desenvolvendo categorias amplas e relações entre elas, o que permitiu identificar temas. 


\section{Resultados}

Pela análise de conteúdo das narrativas dos focus grupo foram identificados como temas principais sobre a intervenção, com a pessoa com DRCT a iniciar hemodiálise, para o processo de adesão: o conceito de adesão; o acolhimento da pessoa; informação e/ou educação, a avaliação e, a promoção de adesão). Dentro de cada tema emergiram categorias como a figura seguinte (Figura 1) ilustra. Saliente-se que em cada categoria estão integradas um número variável de sub-categorias.

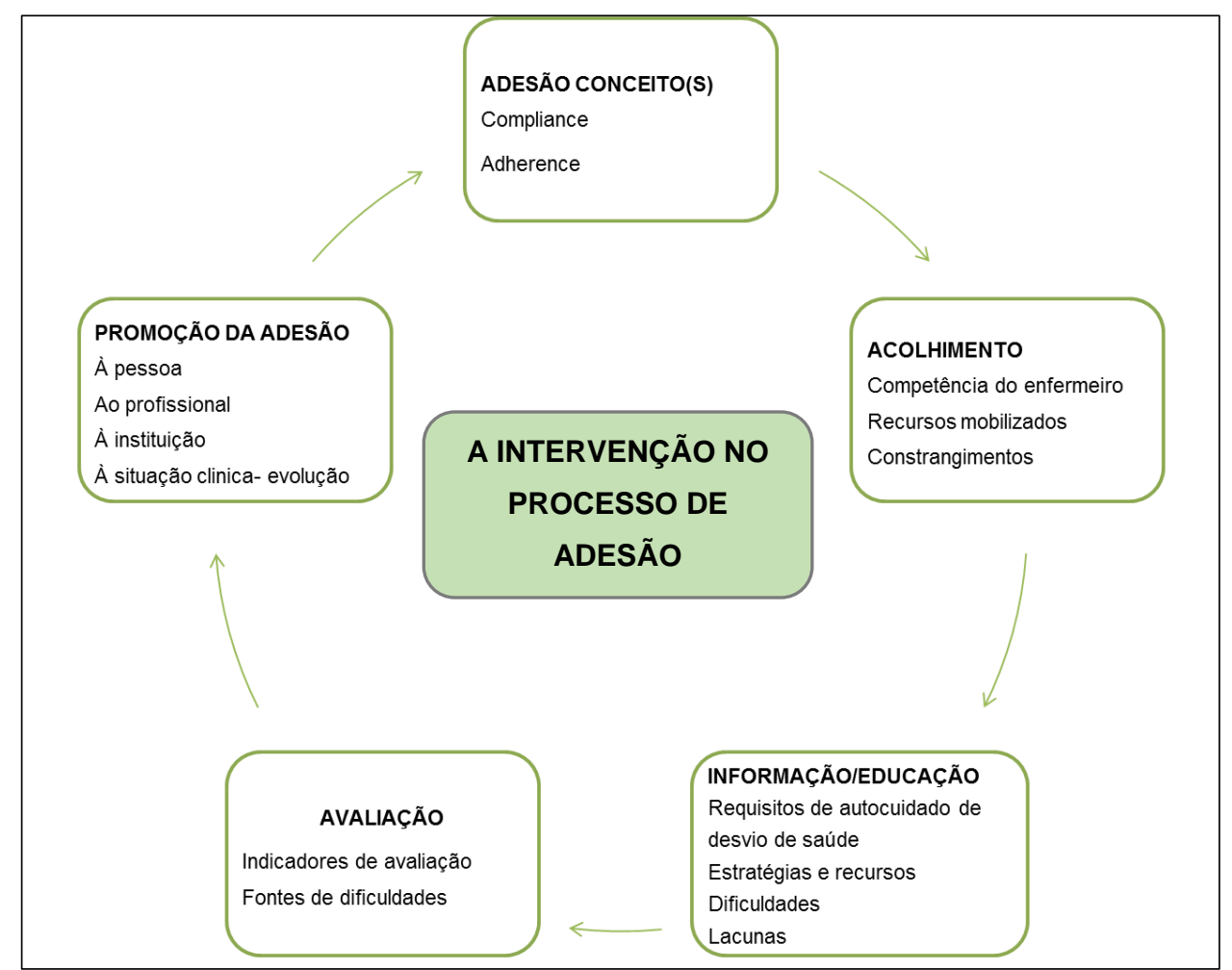

Figura 1: A intervenção do enfermeiro para o processo de adesão da pessoa com DRCT - temas e categorias

Fonte: Adesão ao regime terapêutico da pessoa em início de hemodiálise - intervenção do enfermeiro (FERNANDES; CRUZ, 2017)

\subsection{Adesão Conceito(s)}

Os participantes expressam conceitos de adesão ao RT integráveis nos seus primeiros dois estádios de desenvolvimento contudo não identificam, pelas narrativas, conceitos associados ao estádio de maintenance (em que a pessoa mantém a adesão ao RT de forma autónoma, sem vigilância, no sentido de garantir o seu bem-estar e um estilo de vida saudável). A adesão é assim, concordância ou cumprimento - compliance - por parte da pessoa do que lhe é prescrito. Apresentam um conjunto de indicadores que foram 
integrados nas subcategorias - medicação, nutrição e ingestão hídrica e regime terapêutico, como exemplifica a expressão de um participante.

"Será o cumprimento da pessoa... Ah... sobre a prescrição que lhe é colocada, quer a alimentação, (...) quer a prescrição mesmo de toda a medicação.” FG - D ${ }^{3}$

Têm, também, uma conceção de adherence, entendida como um processo contínuo e gradual, de colaboração mútua entre profissionais e doente, que implica aceitação e transição, tendo a pessoa que dar consentimento e como tal possuir conhecimento e compreensão do RT a que irá ser sujeito para que se opere uma mudança de comportamentos e consequentemente nos hábitos de vida, tal como se ilustra,

(...) a compreensão para a adesão, é preciso ele compreender para cumprir o regime que é benéfico para ele. $\mathrm{FG}-\mathrm{A}$

Com os comportamentos do doente vimos se aderiu ou não aderiu (...) Há uma mudança dos hábitos de vida... $\mathrm{E}$ nós alertamos muito para isso... $\mathrm{FG}-\mathrm{B}$

É a capacidade que ele tem de dar continuidade a esse mesmo tratamento... FG $-\mathrm{C}$

\subsection{Acolhimento}

O acolhimento na unidade é da competência da equipa de enfermagem pelo conhecimento e relação terapêutica que estabelece com a pessoa e pela continuidade de/nos cuidados. No processo há adequação dos recursos mobilizados contudo, não há um padrão seguido pela equipa. É diferenciado de acordo com a pessoa, as suas respostas à situação clínica, as intervenções terapêuticas já realizadas ou o nível de conhecimento sobre a situação de saúde.

\section{(...) ninguém melhor que o enfermeiro sabe aquilo que o doente precisa de saber para fazer o seu tratamento. Conhece a pessoa e conhece aquilo que a pessoa irá passar (...) ninguém melhor que o enfermeiro para conseguir orientar a pessoa... FG - C}

No acolhimento a escassez de dados sobre a situação clínica, o estado de consciência ou outras respostas à situação da pessoa e, a ausência de normas de atuação são constrangimentos identificados.

É um doente que não tem conhecimento, se calhar nesse inicio está confuso e só passados alguns 2 ou 3 dias é que lhe podemos realmente fazer o acolhimento... FG - C

(...) toda a gente faz o acolhimento, mas não existe uma estrutura sistematizada, programada e escrita com os passos que devem ser cumpridos por todos. Não é que não se faça! FG - D

\footnotetext{
${ }^{3}$ FG-D - As letras F e $\mathrm{G}$ pretendem designar focus grupo e a letra D designa uma das entrevistas realizadas.
} 


\subsection{Informação e Educação}

Na promoção da adaptação da pessoa com doença renal em início de hemodiálise, à sua nova situação de saúde, os profissionais destacam que desenvolvem intervenções direccionadas à informação e educação da pessoa, tendo como objetivo a adesão ao regime terapêutico.

A informação adequada aumenta o conhecimento da pessoa conduzindo a uma maior probabilidade de melhorar os comportamentos de adesão e a adoção de estilos de vida saudáveis, potencialmente capacitá-la para o autocuidado. Na preparação da pessoa com doença renal em início de hemodiálise, é importante preparar sobre os tratamentos e os ganhos em saúde, a nutrição e ingestão hídrica, os acessos vasculares, os hábitos de vida e os direitos e deveres, estes são requisitos de autocuidado de desvio de saúde

(...) explicamos de que modo é que o tratamento lhe garante uma melhor qualidade de vida, como vai ter a sintomatologia controlada (...) fazemos muitos ensinos incidindo na alimentação - no potássio, no sódio, no fósforo, etc. $\mathrm{FG}-\mathrm{A}$

São estratégias e recursos utilizados no processo formativo, a educação pelos pares, a consciencialização da pessoa e da sua situação clinica, o envolvimento da família, a rentabilização do tempo, a adequação da metodologia utilizada, o questionamento/diálogo com a pessoa, a consulta de esclarecimento e o recurso a outros profissionais ou redes de apoio, como se exemplifica.

Se ele não estiver disponível a ouvir e a aceitar a informação, é informação que é perdida. (...) o prestador de cuidados no nosso serviço é extremamente importante, devido à faixa etária. FG - A

(...) a partir da hora de almoço podemos aproveitar para mais tempo para os familiares e para estar a conversar com os doentes (...) Muitas vezes temos que olhar para o doente que temos à nossa frente, não é... Não vou falar para uma professora da mesma forma que vou falar para uma doente que tem 80 anos e que só tem a $4^{\mathrm{a}}$ classe, não é? FG - B

No processo de preparação da pessoa com doença renal são identificados um conjunto de aspetos que o condicionam de forma menos positiva, estes foram integrados na categoria - dificuldades. No processo de preparação para o início da HD surgem aspectos que o condicionam de forma menos positiva - dificuldades ligadas à organização dos cuidados, aos profissionais, à pessoa cuidada (como se ilustra abaixo) e ao próprio processo formativo (falta de estruturação e sistematização e, complexidade).

É a privacidade para falarmos á vontade com o doente. Por exemplo, o XXX, ele estava bem informado, mas não gostava de falar ao pé dos outros doentes. FG - B 
Nós cada vez mais temos que estar mais despertos para estas situações, porque cada vez mais vamos sendo confrontados com este tipo de doentes. Doentes cada vez mais informados (...) exige de nós, um conhecimento mais atualizado, (...) é uma área que está sempre em evolução. FG - B

\subsection{Avaliação}

A avaliação do processo formativo por parte dos enfermeiros é fundamental pois é a partir desta que compreendem a evolução da capacitação da pessoa para atingir os objetivos e podem equacionar a repetição ou reforço de alguns aspetos do processo formativo.

A avaliação do processo formativo é fundamental pois, é a partir desta que compreendem a evolução da capacitação da pessoa (a curto e longo prazo) para atingir os objetivos podendo perspetivar repetições ou reforços formativos. Avaliam a partir de indicadores como o conhecimento não demonstrado, a qualidade das respostas, as respostas fisiológicas e, as questões e feedback da pessoa.

\footnotetext{
Quando passamos um tipo de informação a um doente temos que avaliar. Há avaliação logo de seguida, aí nós avaliamos se a pessoa captou a nossa informação logo. E depois temos a avaliação passado algum tempo, que já não é para saber se ela tem a informação, mas é para saber se ela mudou determinados comportamentos e atitudes. Na hemodiálise nós fazemos mais através do diálogo, da observação direta, avalia-se o peso por exemplo (...) Avalio o acesso, se eu disser ao doente para ter cuidado em não molhar o penso, não é? Eu vejo se ele na próxima sessão o traz seco ou não.” FG - D

(...) se ele depois vem com alguma dúvida ou se o vês a ter determinado comportamento, com o feedback dele... Alguma questão, alguma conversa, nós apercebemo-nos. Olha, não interiorizou. Não ficou bem. E voltamos à questão." FG - B
}

Contudo, da análise das narrativas emergiram fontes de dificuldade percebidas pelos enfermeiros na avaliação do processo formativo tais como, duração do internamento, falta de sistematização e estruturação do processo formativo e características da pessoa.

\subsection{Promoção da Adesão}

Os participantes percecionam que, a melhoria dos comportamentos de adesão ao regime terapêutico é promovida a partir de cuidados diferenciados e de uma intervenção multidisciplinar. Salientam como mais relevante na promoção da adesão ao RT aspetos associados à pessoa (conhecimento, personalidade, estado emocional e motivacional e apoio), à evolução da situação clínica (regressão da sintomatologia, consciencialização 
da pessoa), ao profissional (coerência, empatia, empenho, relação terapêutica e disponibilidade) e ao contexto e organização da instituição.

(...) há a subjetividade também dos doentes, há doentes que se sentem de certa forma motivados com o tratamento e investem nele e há outros que de certa forma se resignam com a situação. (....) "os doentes que tem apoio familiar tem maior aderência, têm porque são várias pessoas a ouvirem o mesmo, a trabalhar para o mesmo. Há uma melhor adesão quando o familiar apoia FG - A

(...) a simpatia que nós damos ao doente, se não fores simpático para ele, ele não ouve o que lhe dizes. (...) É desmistificar e esclarecer algumas dúvidas que eles tenham, estabelecer confiança com o doente, comunicação. (...) Pôlos à vontade. Se eles não se sentirem à vontade, com alguém que esteja perto deles, eles também não vão falar connosco. FG - D

Em síntese evidencia-se que a complexidade do regime terapêutico da pessoa com doença renal crónica é um aspeto que influência de forma negativa a adesão terapêutica.

\section{Discussão}

A investigação realizada explora a prática de cuidados, desenvolvida pelos enfermeiros, com a pessoa doente renal crónica em início de tratamento dialítico, uma temática que continua a exigir maior conhecimento (GOOVAERTS et al., 2015). Partindo dos resultados procede-se agora à sua discussão crítica, procurando-se, sempre que possível, uma compreensão integradora e justificada pelo confronto com o quadro teórico de referência e com resultados de outras investigações realizadas no mesmo domínio temático.

O acolhimento da pessoa com doença renal em início de hemodiálise, quando é hospitalizada é da competência do enfermeiro pois, é ele que pela sua intervenção tem um melhor conhecimento da pessoa e do contexto, estabelecendo com ela um elo de ligação, uma relação de proximidade e de presença permanentes. Este é um resultado que vai ao encontro da função dos enfermeiros de nefrologia que Goovaerts et al. (2015) apresentam quando consideram que estes têm maior responsabilidade pelos doentes individuais e, atuam como o principal coordenador de contato e atendimento.

Cada experiência de acolhimento deve ser flexível e ajustada à situação clínica da pessoa, não pode ser padronizado. A escassez de dados sobre a situação clínica, o estado de consciência e outras respostas que a pessoa apresenta decorrentes da situação de doença, a ausência de uma norma orientadora ou de um guia de acolhimento, são constrangimentos ao acolhimento identificados pelos participantes. Estes resultados vão ao encontro dos identificados por Costa e Cambiriba (2010), na sua investigação. 
A adesão é compreendida por alguns dos participantes como compliance, em que aderir é cumprir as indicações prescritas pelos profissionais de saúde. Outros, substituem a conceção anterior por Adherence o que sugere uma diminuição do poder do clínico na decisão do tratamento e, procura o envolvimento numa parceria ativa, pessoa-profissional de saúde. A pessoa e a sua família terão de ter conhecimento sobre o RT, compreendendoo, pois só assim se poderá iniciar o processo de aceitação face às alterações decorrentes da doença e a consequente transição, necessária para adoção de novos comportamentos e hábitos de vida. Este, é um processo que pressupõe continuidade e implicação da pessoa para manter o estado de saúde em colaboração com o profissional.

Estes são resultados que não estão no alinhamento da revisão sistemática de literatura realizada por Alikari, Fradelos e Zyga (2015), que orientam para a relevância dos factores demográficos na adesão da pessoa ao processo terapêutico.

A recuperação da "normalidade" da vida da pessoa/família com doença renal pode ser influenciada pela qualidade da educação terapêutica dos profissionais de saúde.

Evidencia-se uma preocupação unânime no esclarecimento sobre o tratamento e os inerentes ganhos em saúde, o esclarecimento das alterações nutricionais e hídricas, os cuidados com os acessos vasculares e as alterações de vida, principalmente da atividade profissional.

Os participantes ajustam o processo formativo à pessoa e à sua situação clínica proporcionando um ensino gradual e progressivo, partindo da avaliação diagnóstica, no sentido da consciencialização sobre a sua situação clínica, preparação emocional e promoção de conhecimentos. Envolvem a família nesta preparação, dadas as profundas alterações com que a pessoa tem que lidar e os reflexos na vida familiar. Rentabilizam o tempo de contacto com a pessoa, aproveitando os horários das refeições e do tratamento hemodialítico para trabalharem com ela na sua formação. Recorrem à educação pelos pares, a panfletos informativos, a outros profissionais ou redes de apoio na preparação/formação e à consulta de esclarecimento. Autores como Teng et al., (2013) também concluem que a intervenção educacional sobre a doença e tratamento capacita a pessoa a participar ativa e conscientemente na adesão ao seu RT.

Apesar da importância atribuída ao papel educativo, na promoção e desenvolvimento de comportamentos para o autocuidado são identificadas dificuldades no processo, relativas ao ambiente organizacional (condições físicas da unidade, conjuntura atual e políticas de austeridade em saúde com escassez de recursos humanos e sobrecarga de trabalho), ao próprio profissional (falta de disponibilidade mental e 
motivação e a deficiente articulação na equipa multidisciplinar), à pessoa cuidada (desresponsabilização da família no cuidado os seus membros e a disponibilidade da pessoa com doença renal para a aprendizagem) e inerentes ao processo formativo (ações avulsas, isoladas e com lacunas).

Nas recomendações que Sharaf (2016) apresenta, a partir da investigação que realizou, é salientada a importância das técnicas motivacionais usadas no aconselhamento em detrimento de abordagens mais tradicionais de dar informação. $\mathrm{O}$ autor recomenda ainda o recurso a um manual de ensino.

A validação do processo formativo, a partir da avaliação do nível de preparação da pessoa para enfrentar e implementar o seu RT, acontece após a intervenção de ensino e a longo prazo. A primeira, após o ensino, é associada ao questionamento e diálogo com a pessoa sobre o que aprendeu. A segunda, a que acontece a longo prazo, é uma avaliação a partir da manifestação de comportamentos ou expressões de não-adesão por parte da pessoa, tais como: o ganho de peso interdialítico, as alterações nos valores analíticos, os valores da pressão arterial ou a ingestão de líquidos. Situação de algum modo similar foi identificada por Sharaf (2016) embora este autor tenha obtido estes indicadores de adesão a partir da avaliação objectiva de alguns parâmetros fisiológicos. A intervenção da equipa na avaliação, pelo recurso àqueles indicadores, é congruente com os que Tourette-Turgis e Isnard-Bagnis (2013) propõem para avaliar a eficácia dos programas de educação terapêutica nos doentes crónicos.

O nível de conhecimento sobre a doença e RT é entendido pelos participantes como potenciador do comportamento de adesão, assim como, o estado emocional e motivacional em que a pessoa se encontra e o envolvimento da família. Os resultados e conclusões apresentadas por Prezotto e Abreu (2014) e Alikari, et al., (2015) são reforçadas por estes que agora são apresentados. A regressão dos sintomas e a aprendizagem pelo erro associada à experiência de uma situação clínica limite por parte da pessoa, são também identificados como fatores que poderão ser promotores da adesão.

Os resultados indicam que o contexto e a organização dos cuidados influenciam no processo de adesão, tendo o empenho dos profissionais, o interesse, a simpatia e a confiança que se estabelece com a pessoa uma importância preditiva na adesão.

As mudanças são complexas e, acima de tudo, impõem o envolvimento de vários intervenientes contudo, os resultados obtidos espelham que a correta planificação e implementação do processo educativo, mobilizando os recursos adequados, conduzem a uma intervenção direcionada à pessoa, integrando-a de uma forma efetiva no processo de 
cuidados, comprometendo-a com os ganhos em saúde e consequentemente com a adherence e maintenance neste processo.

\section{Conclusão}

A intervenção dos enfermeiros no processo de adesão ao regime terapêutico da pessoa com DRC em início de HD assume-se de particular relevância.

Os enfermeiros reconhecem que são os profissionais mais competentes para acolher a pessoa em início de hemodiálise e estabelecer com ela uma ligação que deve ser uma relação de proximidade e confiança, o que poderá ser determinante para o sucesso de todo o processo.

Os conceitos de adesão são díspares, contudo unânimes na importância atribuída ao envolvimento da pessoa no processo de adesão. A educação terapêutica da pessoa é um processo complexo orientado pelo esclarecimento sobre a terapia dialítica, os ganhos em saúde, mudanças nutricionais e hídricas, cuidados com acessos vasculares e elucidação das alterações de vida a implementar para o sucesso da terapia. As dificuldades identificadas pelos participantes no processo educativo passam por: internamentos com uma curta duração; escassez de recursos humanos que ocasiona sobrecarga de trabalho e consequente limitação de tempo para preparar a pessoa e/ou a família; falta de motivação dos profissionais; articulação na equipa multidisciplinar deficitária; e falta de estruturação e sistematização do processo. No processo de adesão ao RT o envolvimento da família, a regressão dos sintomas, o empenho, a simpatia e confiança estabelecida com a pessoa são considerados aspetos fundamentais.

As conclusões da investigação terão de ser analisadas à luz de factores que as poderão influenciar, por um lado, a colheita de dados ter sido contextualizada a uma unidade de cuidados e, por outro, uma das investigadoras fazer parte da equipa de saúde e, do contexto investigado, o que independentemente dos esforços feitos para manter a maior distância e neutralidade no processo, poderá ter de algum modo condicionado os resultados obtidos.

\section{Referências}

ALIKARI, V. et al. The Effect of Nursing Counseling on Improving Knowledge, Adherence to Treatment and Quality of Life of Patients Undergoing Hemodialysis. International Journal of Caring Science, Grécia, v. 8, n. 2, p. 514-518, may./aug. 2015. 
ALIKARI, V.; FRADELOS, E.; ZYGA, S. Family support, social and demographic correlations of non-adherence among haemodialysis patients. American Journal of Nursing Science, New York, v. 4, n. 2-1, p. 60-65, feb. 2015.

BARBOUR, R. Grupos Focais. 1. ed. Porto Alegre: Artmed, 2009.

BOGDAN, R.; BIKLEN, S. Investigação Qualitativa em Educação: Uma introdução à teoria e aos métodos. 1. ed. Porto: Porto Editora, 2013.

CAREY, M. O efeito do grupo nos grupos de foco: Planear, implementar e interpretar investigação com grupos de focos. In: MORSE, J. (Ed.). Aspetos essenciais de metodologia de investigação qualitativa. Coimbra: Formasau, 2007. p. 223-239.

CATELA, A.; AMENDOEIRA, J. Viver a Adesão ao Regime Terapêutico: Experiências Vividas do Doente Submetido a Transplante Cardíaco. Pensar Enfermagem, Lisboa, v.14, n.2, p. 39-54, dez. 2010.

CHAMNEY, M. (Ed.). Competency framework. Education Board of EDTNA/ERCA and ENRCA. Lucerne: ENRCA, 2007. Disponível em:

<http://www.edtnaerca.org/pdf/education/CompetencyBook.pdf〉. Acesso em: 10 fev. 2017.

COSTA, M.; CAMBIRIBA, M. Acolhimento em enfermagem: A visão do profissional e a expectativa do usuário. Ciência, Cuidado e Saúde, Maringá, v.9, n.3, p. 494-502, jul./set. 2010.

DIREÇÃO GERAL DE SAÚDE (DGS). Tratamento conservador médico da insuficiência renal crónica: Estádio 5. Circular Normativa. Nº 017. 35p. 2011.

DRAWZ, P. et al. Impact of a chronic kidney disease registry and provider education on guideline adherence - a cluster randomized controlled trial. BMC Medical Informatics and Decision Making, London, v. 12, n. 62, p. 1-8, jul. 2012.

FERNANDES, M.; CRUZ, L. Adesão ao regime terapêutico da pessoa em início de hemodiálise - intervenção do enfermeiro. In: CONGRESSO IBERO-AMERICANO EM INVESTIGAÇÃO QUALITATIVA E INTERNATIONAL SYMPOSIUM ON QUALITATIVE RESEARCH, 6, 2017, Salamanca. Anais... Salamanca: LudoMedia, 2017. p. 572-581.

GOOVAERTS, T. et al. Continuing education: preparing patients to choose a renal replacement therapy. Journal of Renal Care, Malden, v. 41, n.1, p. 62-75, mar. 2015.

MACIEL, C. et al. Adesão ao tratamento hemodialítico: percepção dos pacientes renais crônicos. Cogitare Enfermagem, Curitiba, v. 20, n. 3, p. 540-547, jul./sept. 2015.

NUNES, A. A utilização da técnica dos grupos focais na metodologia de investigação qualitativa. Revista investigação em enfermagem, Coimbra, n. 25, p. 89-94, fev. 2012.

PIRES, Á. Amostragem e pesquisa qualitativa: ensaio teórico e metodológico. In : POUPART, J. et al. (Eds.). A Pesquisa qualitativa: Enfoques epistemológicos e metodológicos. 3. ed. Petrópolis: Editora Vozes, 2012. p. 154-211.

POUPART, J. et al. A Pesquisa qualitativa: Enfoques epistemológicos e metodológicos. 3. ed. Petrópolis: Editora Vozes, 2012.

PREZOTTO, K.; ABREU, I. O paciente renal crónico e a adesão ao tratamento hemodialítico. Revista de enfermagem Universidade Federal de Pernambuco, Recife, v. 8, n. 3, p. 600-605, mar. 2014. 
SHARAF, A. The impact of educational interventions on hemodialysis patients' adherence to fluid and sodium restrictions. IOSR Journal of Nursing and Health Science, Ghaziabad, v.5, n.3, p.50-60, may./june. 2016.

TENG, H-L. et al. Effects of targeted interventions on lifestyle modifications of chronic kidney disease patients: Randomized controlled trial. Western Journal of Nursing Research, Columbia, v.35, n.9, p.1107-1127, oct. 2013.

TOURETTE-TURGIS, C.; ISNARD-BAGNIS, C. Éducation thérapeutique - Patient education. Néphrologie \& Thérapeutique, Paris, v. 9, n. 4, p.235-240, july. 2013.

WORLD HEALTH ORGANIZATION (WHO). Global status report on noncommunicable diseases: Attaining the nine global noncommunicable diseases targets; a shared responsibility. Geneva, Switzerland: World Health Organization WHO Press. 2014.

WORLD HEALTH ORGANIZATION (WHO). Noncommunicable Diseases Progress Monitor. Geneva: World Health Organization, 2017.

Recebido em: 25 de março de 2018.

Aceito em: 30 de março de 2018. 